\title{
Evaluation of Health-Related Quality of Life Associated with Provision of Healthcare to Stroke Patients Living at Home in Japan
}

\author{
Sayuri Kaneko1 ${ }^{*}$, Masako Kanekawa ${ }^{2}$ \\ ${ }^{1}$ School of Nursing, Nagoya City University, Nagoya, Japan \\ ${ }^{2}$ Tohoku University Graduate School of Medicine, Sendai, Japan \\ Email: ${ }^{*}$ kaneko@med.nagoya-cu.ac.jp
}

Received 28 July 2015; accepted 12 September 2015; published 15 September 2015

Copyright (C) 2015 by authors and Scientific Research Publishing Inc.

This work is licensed under the Creative Commons Attribution International License (CC BY). http://creativecommons.org/licenses/by/4.0/

(c) (i) Open Access

\section{Abstract}

Objective: As stroke mortality rates decline in Japan, a large proportion of disabled stroke survivors living in their homes are supported by informal caregivers or formal healthcare services. To evaluate the impact of healthcare provision on outcome of stroke patients living at home, this study investigated the associations of long-term care and health-related quality of life (HRQOL) in patients 1 year after stroke onset. Methods: Data on patient and caregiver characteristics, HRQOL of patients, and healthcare services for those living at home were prospectively collected from 426 patients with stroke at baseline and 12 months. Using general measures of HRQOL, namely, Short Form-36 (SF-36) and EuroQOL 5 dimension (EQ-5D), multivariate regression models were used to determine the contribution of variables to changes in HRQOL scores from discharge to the first year after stroke. Results: Five domains of SF-36-role-physical, vitality, social functioning, roleemotional, and mental health-were significantly improved 1 year after stroke. Factors affecting changes in the five domains of HRQOL were age, independence in activities of daily living, and cognitive function. Home care service was positively associated with role-physical, social functioning, and role-emotional. In addition, home rehabilitation and home bathing services were positively associated with social functioning. Conclusion: This study clarified that improvements of HRQOL 1 year after stroke were associated with use of home-based services involving home care service, home rehabilitation, and home bathing services. The use of home-based services contributed to the improved welfare of patients living at home.

\section{Keywords}

Stroke, Health-Related Quality of Life, Long-Term Care, Healthcare

\footnotetext{
${ }^{*}$ Corresponding author.
} 


\section{Introduction}

The number of disabled stroke survivors is increasing with the decline in stroke mortality. Stroke decreases health-related quality of life (HRQOL) of survivors. Many factors have been shown to influence the HRQOL of stroke patients, including age [1]-[3], sex [2] [4]-[6], motor impairment [5] [7] [8], physical disability or dependency in activities of daily living (ADL) [1] [8] [9], cognitive impairment [1] [3], and depression [1] [3] [5] [10]. In addition, a previous study showed that the decline of HRQOL of stroke patients was associated with family care giving due to the increased burden on caregivers [11].

A large proportion of disabled stroke survivors living at home are supported by informal caregivers or formal healthcare services in Japan [12]. The influence of formal healthcare services on the HRQOL of stroke patients is unknown. In Japan, formal healthcare services are covered by the national long-term care insurance system. However, issues related to the care system are becoming apparent; for example, the advanced age of caregivers, the growing burden on caregivers, and the imbalance between supply and demand of long-term care due to an increasing number of patients requiring home care [13].

Most studies on long-term outcomes of stroke focused on patient or caregiver characteristics. It is necessary to examine the effect of provision of care services on patient outcomes based on observations of patient changes after using such services. Previous studies showed the relationship between home care and stroke patient outcome, using outcome measures of changes in the level of care needs [14], functional independence measure (FIM) [9] [15], or ADL [16]. However, no study has examined the relationship between home care services and stroke outcomes using HRQOL. In particular, changes in HRQOL at 1 year after stroke onset have not been clarified in Japan.

The purposes of this study were to evaluate HRQOL associated with provision of healthcare to patients living at home 1 year after stroke and to determine predictors of HRQOL among those patients from discharge to 1 year after stroke.

\section{Methods}

\subsection{Participants and Setting}

A total of 541 participants were included in this prospective cohort study. The study included all patients who were admitted to acute hospitals from January 2009 to December 2013 after experiencing a stroke. All patients met the following inclusion criteria: (1) hospitalized with a diagnosis of an ischemic stroke, hemorrhagic stroke, or transient ischemic attack (TIA); (2) started receiving home care after discharge; and (3) certified by the long-term care insurance system of the Ministry of Health, Labour and Welfare in Japan as requiring care support. No exclusion criteria were applied.

This was a survey of patients living at home who were receiving assistance from any of 42 facilities in Hokkaido, Tohoku, or Kanto-Koshinetsu regions in Japan. Facilities included medical clinics, home care nursing stations, in-home care support centers, rehabilitation institutions, and regional medical centers.

\subsection{Data Collection}

Data collected included patient characteristics, caregiver characteristics, HRQOL of patients, and information on provision of home-based healthcare. Data were collected in two phases: in phase 1, data on HRQOL of patients were collected prospectively via a self-administered questionnaire when the patient began receiving home care and at 1 year after stroke. In phase 2, data on patient characteristics, caregiver characteristics, and provision of home-based healthcare were gathered from medical charts and nursing records by medical staff at 1 year after the onset of stroke.

\subsection{Instruments}

Patient characteristics included age, sex, required level of care that was covered by long-term care insurance, independence in ADL, cognitive function, type of stroke (ischemic, hemorrhagic, or TIA), and number of strokes (first or recurrence). The level of care covered by long term-care insurance was classified into six categories according to the support required and the care level needed. Category 1 represented whether or not support was required, such as support for everyday activities like cooking and house cleaning. The remaining 5 
categories were based on the care level defined by long-term care insurance: level 1 is a state that requires partial care for some aspects of ADL, level 2 requires a low level of care, level 3 requires a moderate level of care, level 4 requires a high level of care, and level 5 is requires the highest level of care.

Caregiver characteristics included age, sex, relationship to the patient, and support from family other than caregivers.

HRQOL of patients was assessed using the Medical Outcome Study 36-Item Short Form Health Survey (SF-36) and the EuroQol 5 Dimension (EQ-5D), which are general HRQOL scales. The SF-36 is a comprehensive QOL scale used to evaluate subjective outcomes, comprising eight domains, each scored from 0 to 100 points: physical functioning; role-physical; bodily pain; general health; vitality; social functioning; role-emotional; and mental health [17]. A higher score indicates higher QOL. In the present study, the SF-36 Japanese version, which has confirmed validity and reliability [18], was used to evaluate HRQOL. The EQ-5D was used to evaluate the following five items on a 3-point scale: mobility; self-care; usual activities; pain/discomfort; and anxiety/depression [19]. The utility score conversion table in the Japanese version of the EQ-5D can be used to calculate QOL utility scores [20]. Higher scores indicate better HRQOL.

Provision of home-based healthcare represented the amount of medical care and long-term care covered by insurance. It was divided into two categories: (1) home-based services, in which medical and care workers visited the home, and (2) institutional care services, in which patients traveled to other facilities. Home-based services included five types of services: a doctor's home visit; home health care provided by a visiting nurse; home care services provided by care helpers; home rehabilitation (e.g., physiotherapy done at home), and home bathing service, performed by care helpers or nurses. Institutional care services included outpatient medicine, outpatient rehabilitation, day-care services, short-stay services, and admission to a hospital.

\subsection{Ethical Approval}

This study was approved by the ethics committees of Tohoku University Graduate School of Medicine and Nagoya City University School of Nursing and was conducted in accordance with institutional guidelines. Each participant received oral and written information about the aim of the study, and it was emphasized that participation was voluntary and would not in any way affect their treatment. All participants provided written informed consent.

\subsection{Statistical Analysis}

First, characteristics of patients who completed follow-up were compared with those who dropped out using the chi-square test.

Second, descriptive statistics and the paired t-test were used to compare the eight domains of the SF-36 and the QOL utility scores of the EQ-5D from discharge to those at 1-year follow-up. This study calculated the change in QOL scores, where a positive change in the score indicated improvement and a negative change indicated deterioration. Then, variables that showed a significant difference, with the level of significance set at $\mathrm{p}<$ 0.05 , in the paired t-test were used as dependent variables and underwent multivariate regression analysis with stepwise selection to determine independent predictors of the change in QOL score. Analyses were performed using SPSS for Windows, Version 20.0J (IBM; Japan), with the level of significance set at $\mathrm{p}<0.05$.

\section{Results}

Of 541 patients and caregivers who were followed, 426 participants with data from onset to 1 year after stroke (follow-up rate, 78.8\%) were analyzed. Among the 115 participants who dropped out during the follow-up period, 52 (45.2\%) did so because they moved, were transferred, or changed offices; 38 (33.0\%) did so for personal reasons; 22 (19.1\%) did so for reasons related to the survey administrators; and 3 (2.6\%) died. No significant differences were noted between those who completed follow-up and those who dropped out in terms of age, sex, and level of care required.

Participant characteristics are shown in Table 1. The mean age of patients was 74.5 years, and 211 (49.5\%) patients were men. A total of 314 (73.7\%) patients experienced their first stroke event. The mean age of caregivers was 66.5 years, 316 (74.2\%) caregivers were women, and 218 (51.2\%) caregivers were a spouse or partner. Table 2 shows the provision of medical care and long-term care covered by insurance services during the 
Patient characteristic

Age, years

$<65$

$65-74$

$\geq 75$

Sex

Male

Female

Level of care required covered by long-term care insurance

Support required

Care Level 1

Care Level 2

Care Level 3

Care Level 4

Care Level 5

Independence in activities of daily living

Can walk independently indoors and outdoors

Can perform self-care independently

Requires partial assistance

Requires full assistance

Cognitive function during everyday activities

No disability/slight disability

Moderate disability

Severe disability

Type of stroke

Ischemic

Hemorrhagic

Transient ischemic attack

Number of strokes

First ever

Recurrence

Caregiver characteristics

Age, years

$<65$

$65-74$

$\geq 75$

Sex

Male

Female

Relationship to patient

Spouse/partner

Children

Relative/friends

Support from other family members

Yes

No 
first year after stroke. The change in HRQOL score in the period from discharge to 1 year after stroke is shown in Table 3. Paired t-test showed that scores for the following SF-36 domains at discharge and after 1 year were significantly different: role-physical $(\mathrm{p}=0.02)$, vitality $(\mathrm{p}<0.01)$, social functioning $(\mathrm{p}<0.01)$, role-emotional $(\mathrm{p}=0.01)$, and mental health $(\mathrm{p}<0.01)$. No significant differences in physical functioning, bodily pain, and general health of the SF-36, and the utility index of EQ-5D were observed.

Table 4 shows the factors associated with HRQOL, highlighting significant differences in five domains of the SF-36 as determined by multiple regression analysis. Age, independence in ADL, and cognitive function were associated with the five domains of SF-36. With respect to care services covered by insurance, role-physical was associated with home care services $(\beta=0.10, \mathrm{p}=0.04)$ and duration of hospitalization $(\beta=-0.15, \mathrm{p}<0.01)$. Vitality was associated with day care services $(\beta=0.12, \mathrm{p}=0.02)$. Social functioning was associated with home

Table 2. Provision of medical care and long-term care covered by insurance.

\begin{tabular}{|c|c|c|c|c|}
\hline & Number of Users & $(\%)$ & Mean Number of Usages per Month & $(\mathrm{SD})$ \\
\hline \multicolumn{5}{|l|}{ Home-based services } \\
\hline Doctor’s home visit & 106 & $(24.9)$ & 1.5 & $(0.7)$ \\
\hline Home health care (provided by nurse) & 124 & $(29.1)$ & 4.8 & (3.9) \\
\hline Home care services (provided by care helper) & 141 & $(33.1)$ & 22.4 & $(17.2)$ \\
\hline Home rehabilitation & 94 & $(22.1)$ & 5.8 & $(3.2)$ \\
\hline Home bathing services & 72 & $(16.9)$ & 4.8 & $(2.4)$ \\
\hline \multicolumn{5}{|l|}{ Institutional care services } \\
\hline Outpatient medicine & 426 & $(100.0)$ & 1.9 & $(2.4)$ \\
\hline Outpatient rehabilitation & 225 & $(52.8)$ & 8.6 & $(4.4)$ \\
\hline Day-care services & 157 & $(36.9)$ & 7.8 & $(3.5)$ \\
\hline Short-stay services, total days per month & 70 & $(16.4)$ & 14.4 & $(7.9)$ \\
\hline Hospitalization, total days per year & 116 & $(27.2)$ & 69.7 & (55.7) \\
\hline
\end{tabular}

SD, standard deviation.

Table 3. Domain scores of short form-36 (SF-36) and EuroQOL 5.

\begin{tabular}{|c|c|c|c|c|}
\hline & Mean Score on Discharge & Mean Score at 1 Year & Difference & p value ${ }^{1}$ \\
\hline \multicolumn{5}{|l|}{ SF-36 } \\
\hline Physical functioning & 19.1 & 22.5 & +3.4 & 0.36 \\
\hline Role-physical & 30.8 & 39.8 & +9.0 & 0.02 \\
\hline Bodily pain & 55.7 & 52.1 & -3.6 & 0.27 \\
\hline General health & 31.6 & 35.6 & +4.0 & 0.18 \\
\hline Vitality & 33.5 & 49.5 & +16.0 & $<0.01$ \\
\hline Social functioning & 27.7 & 50.9 & +23.2 & $<0.01$ \\
\hline Role-emotional & 26.2 & 34.2 & +8.0 & 0.01 \\
\hline Mental health & 33.1 & 54.8 & +25.8 & $<0.01$ \\
\hline \multicolumn{5}{|l|}{ EuroQOL 5} \\
\hline Utility index & 0.304 & 0.470 & +0.098 & 0.41 \\
\hline
\end{tabular}

Positive difference reflects improvement and negative difference indicates deterioration in the period from discharge to 1 year after stroke.

${ }^{1}$ Paired t-test. 
Table 4. Multiple regression analysis of factors associated with health-related quality of life scores.

\begin{tabular}{|c|c|c|c|c|c|c|c|c|c|c|}
\hline \multirow[b]{2}{*}{ Variable } & \multicolumn{2}{|c|}{ Role-physical } & \multicolumn{2}{|c|}{ Vitality } & \multicolumn{2}{|c|}{ Social functioning } & \multicolumn{2}{|c|}{ Role-emotional } & \multicolumn{2}{|c|}{ Mental health } \\
\hline & $\beta$ & $\mathrm{p}$ value & $\beta$ & $\mathrm{p}$ value & $\beta$ & $\mathrm{p}$ value & $\beta$ & $\mathrm{p}$ value & $\beta$ & $\mathrm{p}$ value \\
\hline \multicolumn{11}{|l|}{ Patient characteristic } \\
\hline Age & -0.15 & $<0.01$ & -0.17 & $<0.01$ & -0.09 & 0.04 & -0.12 & $<0.01$ & -0.11 & 0.03 \\
\hline Sex & - & - & - & - & - & - & - & - & - & - \\
\hline Level of care required covered by insurance & -0.13 & 0.02 & - & - & -0.15 & $<0.01$ & -0.12 & 0.03 & - & - \\
\hline Independence in activities of daily living & -0.17 & $<0.01$ & -0.15 & $<0.01$ & -0.19 & $<0.01$ & -0.15 & $<0.01$ & -0.11 & 0.02 \\
\hline Cognitive function during everyday activities & -0.14 & $<0.01$ & -0.14 & $<0.01$ & -0.12 & $<0.01$ & -0.13 & $<0.01$ & -0.11 & 0.02 \\
\hline Type of stroke & - & - & - & - & - & - & - & - & - & - \\
\hline Number of strokes & - & - & - & - & - & - & - & - & - & - \\
\hline \multicolumn{11}{|l|}{ Caregiver characteristic } \\
\hline Age & - & - & - & - & - & - & - & - & - & - \\
\hline Sex & - & - & - & - & - & - & - & - & - & - \\
\hline Relationship to patient & - & - & - & - & - & - & - & - & - & - \\
\hline Support from other family members & - & - & - & - & - & - & - & - & - & - \\
\hline \multicolumn{11}{|l|}{ Provide medical care and long-term care } \\
\hline Doctor’s home visit & - & - & - & - & - & - & - & - & - & - \\
\hline Home health care (provided by nurse) & - & - & - & - & - & - & -0.09 & 0.04 & - & - \\
\hline Home care services (provided by care helper) & 0.10 & 0.04 & - & - & 0.12 & $<0.01$ & 0.09 & 0.04 & - & - \\
\hline Home rehabilitation & - & - & - & - & 0.09 & 0.03 & - & - & - & - \\
\hline Home bathing services & - & - & - & - & 0.13 & $<0.01$ & - & - & - & - \\
\hline Outpatient medicine & - & - & - & - & - & - & - & - & - & - \\
\hline Outpatient rehabilitation & - & - & - & - & - & - & - & - & - & - \\
\hline Day-care services & - & - & 0.12 & 0.02 & 0.16 & $<0.01$ & - & - & 0.20 & $<0.01$ \\
\hline Short-stay services & - & - & - & - & -0.21 & $<0.01$ & - & - & - & - \\
\hline Hospitalization & -0.15 & $<0.01$ & - & - & -0.18 & $<0.01$ & -0.15 & $<0.01$ & - & - \\
\hline $\mathrm{R}$ & \multicolumn{2}{|c|}{0.40} & \multicolumn{2}{|c|}{0.34} & \multicolumn{2}{|c|}{0.46} & \multicolumn{2}{|c|}{0.47} & \multicolumn{2}{|c|}{0.31} \\
\hline Adjusted $\mathrm{R}^{2}$ & \multicolumn{2}{|c|}{0.16} & \multicolumn{2}{|c|}{0.11} & \multicolumn{2}{|c|}{0.21} & \multicolumn{2}{|c|}{0.22} & \multicolumn{2}{|c|}{0.10} \\
\hline
\end{tabular}

$\beta$, standardized coefficient by stepwise linear regression analysis.

care services $(\beta=0.12, \mathrm{p}<0.01)$, home rehabilitation $(\beta=0.09, \mathrm{p}=0.03)$, home bathing services $(\beta=0.13, \mathrm{p}<$ $0.01)$, day-care services $(\beta=0.16, \mathrm{p}<0.01)$, short-stay services $(\beta=-0.21, \mathrm{p}<0.01)$, and duration of hospitalization $(\beta=-0.18, \mathrm{p}<0.01)$. Role-emotional was associated with home health care $(\beta=-0.09, \mathrm{p}=0.04)$, home care services $(\beta=0.09, \mathrm{p}=0.04)$, and duration of hospitalization $(\beta=-0.15, \mathrm{p}<0.01)$. Mental health was associated with day care services $(\beta=0.20, \mathrm{p}<0.01)$.

\section{Discussion}

This study clarified two important points. First, the study examined the HRQOL of patients living at home 1 year after stroke. Second, the study determined independent predictors of changes in HRQOL from discharge to 
1 year after stroke.

First, the five domains of SF-36 (role-physical, vitality, social functioning, role-emotional, and mental health) were significantly improved 1 year after stroke onset. These domains are known to involve mental function in general [18]. Javier (2000) showed that QOL and depression were improved 1 year after stroke onset [5]. Results in the present study agree with that study and support the idea that improvement in mental function after stroke is expected. Participants in this study maintained good cognitive function, although their mean age was relatively high. This was likely influenced by the fact that this group was limited to patients who could answer questions about HRQOL, so they all had a relatively higher level of cognitive function. Therefore, patients with good cognitive function are likely to experience improvement of the five domains of SF-36 related to mental function within 1 year after stroke.

In contrast, the SF-36 (physical functioning, bodily pain, and general health) and the EQ-5D utility index remained unchanged from discharge to the first year. Physical functioning and bodily pain of SF-36 are related to physical function, as these domains are correlated strongly with FIM [9]. Previous studies on the ADL of patients discharged from the acute care unit and recovery rehabilitation center have shown a decrease in functional independence and activity level in the first year after stroke [21]. Studies have also shown that getting an adequate amount of exercise through activities such as indoor walking can mitigate the decrease in ADL [15]. However, other findings have indicated decreases in physical function after 3 months [4] [22] and have shown that patients have difficulty maintaining physical function for 6 months [23] [24].

The possible reason for the observed insignificant difference in physical functioning and bodily pain might be that any improvement in these domains would only be seen around the first 3 months after stroke onset. That is, improvement in the physical functions of stroke patients at 3 months or more was not very likely; thus, no difference was seen at 1 year after stroke. An additional reason for no significant difference in general health of SF-36 and utility index of EQ-5D in this study was that these measurements encompass both physical and mental functions [9].

In this study, independent predictors of the change in HRQOL for the five SF-36 domains of role-physical, vitality, social functioning, role-emotional, and mental health from discharge to 1 year after stroke were age, independence in ADL, and cognitive function. That is, advanced age, dependency in ADL, and worse cognitive function were associated with decreased HRQOL. These results are in agreement with previous studies [5] [6] [8] [23] [25]. The present study found no correlations between the HRQOL of patients and caregiver characteristics, although the survey items in this study were limited. This study also found that medical care and long-term care covered by insurance affected each of the five domains differently. With home-based services, the home care service was positively associated with role-physical, social functioning, and role-emotional, while home rehabilitation and home bathing services were positively associated with social functioning. Home care, home rehabilitation, or home bathing services enable the patient to maintain a certain degree of independence in society, because of help from a third party [14] [26]. In contrast, home health care was negatively associated with role-emotional. It is possible that the use of home health care indicated that symptoms had been aggravated, leading to the use of home healthcare by a visiting nurse rather than home care service by a care helper. Hospitalization was also negatively associated with role-physical, social functioning, and role-emotional. This might be related to the lower HRQOL of patients in a poor state of health who required hospital admission or care from a visiting nurse. In institutional care services, day-care service was positively associated with vitality, social functioning, and mental health. The day service programs provide basic services run by care workers including meals, transportation, bathing, and recreation [13]. It is believed that these activities involving contact with care workers lead to physical activity of patients and contribute to their vitality. On the other hand, short-stay services were negatively associated with social functioning. Users of short-stay services were less independent in ADL or had worse cognitive impairment than non-users [13]. However, this service may reduce the burden of caregivers, helping them continue to provide care [13]. Therefore, similar to hospitalization, more frequent use of this service was associated with lower HRQOL of patients.

The present study has several limitations. First, the results of the present study are based on a survey conducted at 42 facilities that provided home-based services. Some stroke survivors were in a hospital or had been admitted into a nursing home. In addition, there may be stroke patients who do not use the long-term care insurance, resulting in selection bias. Thus, the change in HRQOL from discharge to 1 year after stroke seen in this patient population cannot necessarily be generalized to other stroke patients. Second, the data did not contain any information regarding the disease history of patients (such as fracture, severe osteoporosis, osteoarthrosis, 
depression, advanced cancer, etc.), the socioeconomic situation, or the care burden of caregivers. Those data may affect the results of the assessment. Our analysis did not include data on informal care provided by the patient's family, which may have an effect on the HRQOL of stroke patients. Third, this study lacked a control group.

\section{Conclusion}

This study clarified that the five domains of SF-36-namely, role-physical, vitality, social functioning, roleemotional, and mental health - were improved 1 year after stroke onset in patients who were at home. The study also found that factors influencing the change in HRQOL 1 year after stroke were age, independence in ADL, and cognitive function. In regard to home-based services, home care service was positively associated with role-physical, social functioning, and role-emotional, while home rehabilitation and home bathing services were positively associated with social functioning. These findings suggest that the use of home-based services contributes to the improved welfare of stroke patients in society.

\section{Sources of Funding}

This study was supported by Japan Society for the Promotion of Science KAKENHI grant number 23659249.

\section{Disclosure}

The authors have not potential conflicts of interest to disclose.

\section{References}

[1] Haacke, C., Althaus, A., Spottke, A., Siebert, U., Back, T. and Dodel, R. (2006) Long-Term Outcome after Stroke Evaluating Health-Related Quality of Life Using Utility Measurements. Stroke, 37, 193-198. http://dx.doi.org/10.1161/01.STR.0000196990.69412.fb

[2] Gray, L.J., Sprigg, N., Bath, P.M.W., Boysen, G., De Deyn, P.P., Leys, D., O’Neill, D. and Ringelstein, E.B., for the TAIST Investigators (2007) Sex Differences in Quality of Life in Stroke Survivors Data from the Tinzaparin in Acute Ischaemic Stroke Trial (TAIST). Stroke, 38, 2960-2964. http://dx.doi.org/10.1161/STROKEAHA.107.488304

[3] Jönsson, A.-C., Lindgren, I., Hallström, B., Norrving, B. and Lindgren, A. (2005) Determinants of Quality of Life in Stroke Survivors and Their Informal Caregivers. Stroke, 36, 803-808. http://dx.doi.org/10.1161/01.STR.0000160873.32791.20

[4] Gargano, J.W. and Reeves, M.J., for the Paul Coverdell National Acute Stroke Registry Michigan Prototype Investigators (2007) Sex Differences in Stroke Recovery and Stroke-Specific Quality of Life Results from a Statewide Stroke Registry. Stroke, 38, 2541-2548. http://dx.doi.org/10.1161/STROKEAHA.107.485482

[5] Carod-Artal, J., Egido, J.A., González, J.L. and de Seijas, E.V. (2000) Quality of Life among Stroke Survivors Evaluated 1 Year after Stroke Experience of a Stroke Unit. Stroke, 31, 2995-3000.

http://dx.doi.org/10.1161/01.STR.31.12.2995

[6] Gall, C., Franke, G.H. and Sabel, B.A. (2010) Vision-Related Quality of Life in First Stroke Patients with Homonymous Visual Field Defects. Health and Quality of Life Outcomes, 8, 33. http://www.hqlo.com/content/8/1/33 http://dx.doi.org/10.1186/1477-7525-8-33

[7] van Exel, N.J., Scholte op Reimer, W.J. and Koopmanschap, M.A. (2004) Assessment of Post-Stroke Quality of Life in Cost-Effectiveness Studies. The Usefulness of the Barthel Index and the EuroQoL-5D. Quality of Life Research, 13, 427-433. http://dx.doi.org/10.1023/B:QURE.0000018496.02968.50

[8] Patel, M.D., Tilling, K., Lawrence, E., Rudd, A.G., Wolfe, C.D.A. and Mckevitt, C. (2006) Relationships between Long-Term Stroke Disability, Handicap and Health-Related Quality of Life. Age Ageing, 35, 273-279. http://dx.doi.org/10.1093/ageing/afj074

[9] Takemasa, S., Nakagoshi, R., Murakami, M., Uesugi, M., Inoue, Y., Gotou, M. and Naruse, S. (2014) Factors Affecting Quality of Life of the Homebound Elderly Hemiparetic Stroke Patients. Journal of Physical Therapy Science, 26, 301-303. http://dx.doi.org/10.1589/jpts.26.301

[10] Pan, J.H., Song, X.Y., Lee, S.Y. and Kwok, T. (2008) Longitudinal Analysis of Quality of Life for Stroke Survivors Using Latent Curve Models. Stroke, 39, 2795-2802. http://dx.doi.org/10.1161/STROKEAHA.108.515460

[11] Kitahama, S., Takemasa, S. and Shimada, T. (2003) Effect of Long-Term Care Insurance on Psychological States of Its Clients and Care-Burden of Their Caregivers. Bulletin of Faculty of Health Sciences Kobe University School of Medicine, 19, 15-24. 
[12] Cabinet Office Government of Japan (2013) White Paper on Aging Society in 2013. http://www8.cao.go.jp/kourei/whitepaper/w-2013/zenbun/index.html

[13] Health Labour and Welfare Statistics Association (2014) Trend of Long-Term Healthcare and Welfare. Journal of Health and Welfare Statistics, 61, 141-167.

[14] Kim, J.N. and Shiwaku, K. (2012) The Effect of Utilization of In-Home Services and the Changes in Levels of Care Needs of Frail Persons (2002-2004). Results of a Two-Year Follow-Up Study. Journal of Rural Medicine, 7, 6-14. http://dx.doi.org/10.2185/jrm.7.6

[15] Suzuki, I., Yanagi, H. and Tomura, S. (2007) A Study of Factors Related to Activities of Daily Living of the Elderly Receiving In-Home Service Longitudinal Study Using Functional Independence Measures. Japanese Society of Public Health, 54, 81-88.

[16] Kono, A. and Kanagawa, K. (2000) Functional Changes in One-Year and Related Factors among Community-Dwelling Frail Elderly. Japanese Society of Public Health, 47, 508-516.

[17] de Vries, G.E., Jorritsma, W., Dijkstra, P.U., Geertzen, J.H.B. and Reneman, M.F. (2014) The Construct Validity of the Short Form-36 Health Survey for Patients with Nonspecific Chronic Neck Pain. International Journal of Rehabilitation Research, 38, 137-143. http://dx.doi.org/10.1097/MRR.0000000000000102

[18] Fukuhara, S. and Suzukamo, Y. (2004) Manual of SF-36v2 Japanese Version. Institute for Health Outcome \& Process Evaluation Research, Kyoto.

[19] The EuroQol Group (1990) EuroQol-A New Facility for the Measurement of Health-Related Quality of Life. Health Policy, 16, 199-208. http://dx.doi.org/10.1016/0168-8510(90)90421-9

[20] Tsuchiya, A., Ikeda, S., Ikegami, N., Nishimura, S., Sakai, I. and Fukuda, T. (2002) Estimating an EQ-SD Population Value Set. The Case of Japan. Health Economics, 11, 341-353. http://dx.doi.org/10.1002/hec.673

[21] Chijiiwa, N., Miyagawa, T., Tokuhara, N., Suenaga, H., Imamura, Y. and Takemasa, S. (2002) Sequential Changes of ADL and Factors Affecting Them in the Homebound Post-Stroke Residual Disabled. Bulletin of Faculty of Health Sciences Kobe University School of Medicine, 18, 1-12.

[22] Malone, M., Hill, A. and Smith, G. (2002) Three-Month Follow Up of Patients Discharged from a Geriatric Day Hospital. Age and Ageing, 31, 471-475. http://dx.doi.org/10.1093/ageing/31.6.471

[23] Wilma, M. and Jane, V. (2003) Quality of Life during and after Inpatient Stroke Rehabilitation. Stroke, 34, 801-805. http://dx.doi.org/10.1161/01.STR.0000057978.15397.6F

[24] Suzukawa, M., Shimada, H., Watanabe, S., Kobayashi, K. and Suzuki, T. (2011) The Relationship between Physical Performances and ADLs Decline during 6 Months in Frail Elderly People Utilizing Long-Term Care Insurance. Rigakuryohogaku, 38, 10-16.

[25] Jonathan, W.S., Richard, H.O., Helen, M.D., Geoffrey, A.D., Richard, A.L.M. and Amanda, G.T. (2002) Brief Comprehensive Quality of Life Assessment after Stroke: The Assessment of Quality of Life Instrument in the North East Melbourne Stroke Incidence Study (NEMESIS). Stroke, 33, 2888-2894. http://dx.doi.org/10.1161/01.STR.0000040407.44712.C7

[26] Makiseko, H., Abe, T., Onuma, T. and Shimada, H. (2009) Factors Associated with Continuous Home Care Services and Effects of Home-Visit Rehabilitations Service in Older Individuals Requiring Home Care: Cluster Randomization trial. Rigakuryohougaku, 36, 382-388. 